\title{
The transmission properties of elastic waves through multilayers of spheres with planar defects
}

Received: 29 November 2014 / Revised: 11 May 2015 / Published online: 28 August 2015

(C) Springer-Verlag Wien 2015

\begin{abstract}
The work presented in this paper discusses the transmission properties of elastic waves through multilayers of spheres which are periodically arranged in a homogeneous host material. The multilayers of spheres can have different kinds of planar defects. These defects are formed by removing one layer of spheres or by changing the radiuses or the material of scatterers in some layers. First, the reflection and transmission matrices of a single layer of spheres are obtained by the multiple scattering approaches and then the reflection and transmission matrices of multilayers of spheres are derived based on the polymerization method. Numerical examples are presented for 15 layers of spheres with one central planar defect layer or two symmetrically arranged planar defect layers. The influences of these planar defects on the frequency-dependent transmission curves are discussed. It is observed that the band gap can be widened evidently by introducing the planar defect, and the defect states appear in the band gap consequently. So the elaborately arranged planar defects are important in designing specific acoustic filters.
\end{abstract}

\section{Introduction}

The elastic wave propagation problem through three-dimensional periodic composites formed by arranging spherical scatterers periodically in a homogeneous host matrix with infinite extension has been studied in recent years [1-5]. Compared with the elastic wave propagation through random composites with random distributed spherical scatterers in host material [6-8], there are unique band bap effects in the frequency domain for the periodic composites. However, the band gap structure is mainly determined by the shape and distribution of scatterers and the material mismatch between the scatterers and the host. How to adjust the width and midfrequency of band gaps when these factors of components are fixed is therefore interesting. Defects that result in the breakage of periodicity in periodic composites have attracted considerable attention due to their evident influences on band gaps and their potential application in designing functional sound equipment such as new acoustical filters and waveguide devices. Point, linear and planar defects are three types of defects classified in terms of their dimensionality. Defects in a one-dimensional composite are shaped by locally changing the periodicity of laminar periodical structures, and they can bring about resonance humps in the original band gap areas. In other word, the introduction of defects in the perfect periodical structure leads to narrow frequency transmission bands within the forbidden band, namely defect states [9-11]. Compared with the defects in the periodical laminated structure, the defects in two-dimensional periodical composite material have multifarious forms and lead to richer physical phenomena. Sigalas used the plane wave expansion method to study the

Q. Zhao · P. J. Wei $(\bowtie)$

Department of Applied Mechanics, University of Science and Technology Beijing, Beijing 100083, China

E-mail:weipj@ustb.edu.cn

P. J. Wei · Q. Tang

State Key Laboratory of Nonlinear Mechanics (LNM), Chinese Academy of Science, Beijing, Beijing 100080, China 
propagation of acoustical waves through two-dimensional periodic composites with point and linear defects $[12,13]$. It is found that defects in those structures create localized states inside the band gaps, and it is suggested that line defects can act as a waveguide for acoustical waves, while point defects can be used as acoustical filters. Torres et al. [14] discussed also the surface states and localization phenomena in linear and point defects. Kafesaki et al. $[15,16]$ mainly studied the wave guides in elastic wave band gap materials by using the finite difference time domain method. By decoupling the longitudinal and transverse vibrations in a solid host, Wu et al. [17-19] studied the point defect states in two-dimensional arrays of water cylinders in a mercury host. However, the influences of defects in the three-dimensional composite on the band gap of a periodic structure have been less studied up to now. Chandra et al. [20] studied the propagation of acoustical waves along the waveguides existing in three-dimensional composites of spherical lead inclusions. The waveguide structures were constructed by removing a row of inclusions from the perfect crystal along some chosen crystallographic direction. Psarobas et al. [21] studied the effect of planar defects in three-dimensional composite of spherical scatterers on the transmission spectrum. It was found that a plane of impurity spheres introduces modes of vibration localized in this plane at certain frequencies within a band gap of a pure phononic crystal. These modes of vibration show up as sharp resonances in the transmittance curves of elastic waves incident on a slab of the crystal. However, only one type of planar defect, namely the radius of spheres in one layer being smaller than in the other, was considered.

In this paper, the transmission behavior of incident elastic waves through periodically arranged multilayers of spheres with different kinds of planar defects is discussed. These planar defects are separately formed by changing the radiuses and the material of spheres in one layer or by removing one layer of spheres from the periodic composites with multiple layers of spheres. Moreover, not only one planar defect layer located in the middle of a slab but also two planar defect layers arranged symmetrically are studied. The transmission coefficients are calculated for one planar defect and two planar defects, respectively. The influences of planar defects on the band gaps and the defect states appearing in the original band gap are discussed based on the numerical results.

\section{Transmission coefficient through multilayers of spheres}

The reflection and transmission coefficients of a single layer of spheres have been studied in a previous work [22]. Here, a short review is given. When an incident plane wave impinges a layer of spheres embedded in a homogeneous isotropic medium, the incident wave and the scattered wave can be expressed in the form of plane waves as

$$
\begin{aligned}
& \boldsymbol{u}^{i n \pm}(\boldsymbol{r})=\boldsymbol{u}_{\alpha}^{i n}(\boldsymbol{r})+\boldsymbol{u}_{\beta}^{i n}(\boldsymbol{r})=\sum_{\mathrm{g}}\left[\boldsymbol{U}_{\alpha \mathrm{g}}^{i n \pm} e^{i \boldsymbol{k}_{\alpha \mathrm{g}}^{ \pm} \cdot \boldsymbol{r}}+\boldsymbol{U}_{\beta \mathrm{g}}^{i n \pm} e^{i \boldsymbol{k}_{\beta \mathrm{g}}^{ \pm} \cdot \boldsymbol{r}}\right] \\
& \boldsymbol{u}^{s c \pm}(\boldsymbol{r})=\boldsymbol{u}_{\alpha}^{s c}(\boldsymbol{r})+\boldsymbol{u}_{\beta}^{s c}(\boldsymbol{r})=\sum_{\mathbf{g}}\left[\boldsymbol{U}_{\alpha \mathrm{g}}^{s c \pm} e^{i \boldsymbol{k}_{\alpha}^{ \pm} \mathrm{g} \cdot \boldsymbol{r}}+\boldsymbol{U}_{\beta \mathrm{g}}^{s c \pm} e^{i \boldsymbol{k}_{\beta}^{ \pm} \mathrm{g} \cdot \boldsymbol{r}}\right]
\end{aligned}
$$

where the sign $+(-)$ indicates the propagation direction of the plane waves from left (right) to right (left); $\boldsymbol{u}_{\alpha}(\boldsymbol{r})$ and $\boldsymbol{u}_{\beta}(\boldsymbol{r})$ represent the displacement fields of the longitudinal and transverse plane elastic waves, respectively; $\mathbf{g}$ is the reciprocal lattice vector; and $\boldsymbol{k}$ is the wave vector.

The expansion coefficients of the scattered waves and the incident wave can be related by

$$
\begin{aligned}
& {\left[\begin{array}{l}
\boldsymbol{U}_{\alpha}^{s c+} \\
\boldsymbol{U}_{\beta}^{s c+}
\end{array}\right]=\left[\begin{array}{ll}
\boldsymbol{M}_{\alpha \alpha}^{++} & \boldsymbol{M}_{\alpha \beta}^{++} \\
\boldsymbol{M}_{\beta \alpha}^{++} & \boldsymbol{M}_{\beta \beta}^{++}
\end{array}\right]\left[\begin{array}{l}
\boldsymbol{U}_{\alpha}^{i n+} \\
\boldsymbol{U}_{\beta}^{i n+}
\end{array}\right]+\left[\begin{array}{ll}
\boldsymbol{M}_{\alpha \alpha}^{+-} & \boldsymbol{M}_{\alpha \beta}^{+-} \\
\boldsymbol{M}_{\beta \alpha}^{+-} & \boldsymbol{M}_{\beta \beta}^{+-}
\end{array}\right]\left[\begin{array}{l}
\boldsymbol{U}_{\alpha}^{i n-} \\
\boldsymbol{U}_{\beta}^{i n-}
\end{array}\right],} \\
& {\left[\begin{array}{l}
\boldsymbol{U}_{\alpha}^{s c-} \\
\boldsymbol{U}_{\beta}^{s c-}
\end{array}\right]=\left[\begin{array}{ll}
\boldsymbol{M}_{\alpha \alpha}^{-+} & \boldsymbol{M}_{\alpha \beta}^{-+} \\
\boldsymbol{M}_{\beta \alpha}^{-+} & \boldsymbol{M}_{\beta \beta}^{-+}
\end{array}\right]\left[\begin{array}{l}
\boldsymbol{U}_{\alpha}^{i n+} \\
\boldsymbol{U}_{\beta}^{i n+}
\end{array}\right]+\left[\begin{array}{ll}
\boldsymbol{M}_{\alpha \alpha}^{--} & \boldsymbol{M}_{\alpha \beta}^{--} \\
\boldsymbol{M}_{\beta \alpha}^{-} & \boldsymbol{M}_{\beta \beta}^{-}
\end{array}\right]\left[\begin{array}{l}
\boldsymbol{U}_{\alpha}^{i n-} \\
\boldsymbol{U}_{\beta}^{i n-}
\end{array}\right] .}
\end{aligned}
$$

The first terms in the right hand sides of Eqs. (3a) and (3b) represent the transmission amplitude and the reflection amplitude when the incident wave comes from the left side of the layer of spheres; the second terms represent the reflection amplitude and the transmission amplitude when the incident wave comes from the right side of the layer of spheres. For the explicit expressions of the matrix $\boldsymbol{M}_{i j}^{p q}$ refer to [20]. 
It is noted that all plane wave expansions, including the incident waves and the scattered waves, are relative to the central scatterer in the layer. Therefore, the total displacement amplitudes at the plane $\mathrm{z}=0^{-}$(side 1 ) and at the plane $\mathrm{z}=0^{+}$(side 2) are, respectively,

$$
\begin{aligned}
& {\left[\begin{array}{l}
\boldsymbol{U}_{\alpha}^{+}(1) \\
\boldsymbol{U}_{\beta}^{+}(1)
\end{array}\right]=\left[\begin{array}{l}
\boldsymbol{U}_{\alpha}^{i n+} \\
\boldsymbol{U}_{\beta}^{i n+}
\end{array}\right],} \\
& {\left[\begin{array}{l}
\boldsymbol{U}_{\alpha}^{-}(1) \\
\boldsymbol{U}_{\beta}^{-}(1)
\end{array}\right]=\left[\begin{array}{l}
\boldsymbol{U}_{\alpha}^{s c-} \\
\boldsymbol{U}_{\beta}^{s c-}
\end{array}\right]+\left[\begin{array}{l}
\boldsymbol{U}_{\alpha}^{i n-} \\
\boldsymbol{U}_{\beta}^{i n-}
\end{array}\right],} \\
& {\left[\begin{array}{l}
\boldsymbol{U}_{\alpha}^{-}(2) \\
\boldsymbol{U}_{\beta}^{-}(2)
\end{array}\right]=\left[\begin{array}{l}
\boldsymbol{U}_{\alpha}^{i n-} \\
\boldsymbol{U}_{\beta}^{i n-}
\end{array}\right],} \\
& {\left[\begin{array}{l}
\boldsymbol{U}_{\alpha}^{+}(2) \\
\boldsymbol{U}_{\beta}^{+}(2)
\end{array}\right]=\left[\begin{array}{l}
\boldsymbol{U}_{\alpha}^{s c+} \\
\boldsymbol{U}_{\beta}^{s c+}
\end{array}\right]+\left[\begin{array}{l}
\boldsymbol{U}_{\alpha}^{i n+} \\
\boldsymbol{U}_{\beta}^{i n+}
\end{array}\right] .}
\end{aligned}
$$

Substitution of Eqs. (3) into (5) and Eq. (7) leads to

$$
\begin{aligned}
& {\left[\begin{array}{c}
\boldsymbol{U}_{\alpha}^{-}(1) \\
\boldsymbol{U}_{\beta}^{-}(1)
\end{array}\right]=\left[\begin{array}{cc}
\boldsymbol{M}_{\alpha \alpha}^{-+} & \boldsymbol{M}_{\alpha \beta}^{-+} \\
\boldsymbol{M}_{\beta \alpha}^{-+} & \boldsymbol{M}_{\beta \beta}^{-+}
\end{array}\right]\left[\begin{array}{c}
\boldsymbol{U}_{\alpha}^{+}(1) \\
\boldsymbol{U}_{\beta}^{+}(1)
\end{array}\right]+\left[\begin{array}{cc}
\boldsymbol{I}+\boldsymbol{M}_{\alpha \alpha}^{--} & \boldsymbol{M}_{\alpha \beta}^{--} \\
\boldsymbol{M}_{\beta \alpha}^{-\alpha} & \boldsymbol{I}+\boldsymbol{M}_{\beta \beta}^{--}
\end{array}\right]\left[\begin{array}{c}
\boldsymbol{U}_{\alpha}^{-}(2) \\
\boldsymbol{U}_{\beta}^{-}(2)
\end{array}\right],} \\
& {\left[\begin{array}{c}
\boldsymbol{U}_{\alpha}^{+}(2) \\
\boldsymbol{U}_{\beta}^{+}(2)
\end{array}\right]=\left[\begin{array}{cc}
\boldsymbol{I}+\boldsymbol{M}_{\alpha \alpha}^{++} & \boldsymbol{M}_{\alpha \beta}^{++} \\
\boldsymbol{M}_{\beta \alpha}^{++} & \boldsymbol{I}+\boldsymbol{M}_{\beta \beta}^{++}
\end{array}\right]\left[\begin{array}{c}
\boldsymbol{U}_{\alpha}^{+}(1) \\
\boldsymbol{U}_{\beta}^{+}(1)
\end{array}\right]+\left[\begin{array}{ll}
\boldsymbol{M}_{\alpha \alpha}^{+-} & \boldsymbol{M}_{\alpha \beta}^{+-} \\
\boldsymbol{M}_{\beta \alpha}^{+-} & \boldsymbol{M}_{\beta \beta}^{+-}
\end{array}\right]\left[\begin{array}{c}
\boldsymbol{U}_{\alpha}^{-}(2) \\
\boldsymbol{U}_{\beta}^{-}(2)
\end{array}\right] .}
\end{aligned}
$$

If the side 1 and side 2 are used to indicate the planes at $z=-a$ and $z=+a$, respectively, Eqs. (8) and (9) should be replaced by

$$
\begin{aligned}
& {\left[\begin{array}{l}
\boldsymbol{U}_{\alpha}^{-}(1) \\
\boldsymbol{U}_{\beta}^{-}(1)
\end{array}\right]=\left[\begin{array}{ll}
\mathcal{Q}_{\alpha \alpha}^{-+} & \mathcal{Q}_{\alpha \beta}^{-+} \\
\mathcal{Q}_{\beta \alpha}^{-+} & \mathcal{Q}_{\beta \beta}^{-\beta}
\end{array}\right]\left[\begin{array}{l}
\boldsymbol{U}_{\alpha}^{+}(1) \\
\boldsymbol{U}_{\beta}^{+}(1)
\end{array}\right]+\left[\begin{array}{ll}
\mathcal{Q}_{\alpha \alpha}^{--} & \mathcal{Q}_{\alpha \beta}^{--} \\
\mathcal{Q}_{\beta \alpha}^{--} & \mathcal{Q}_{\beta \beta}^{-\beta}
\end{array}\right]\left[\begin{array}{c}
\boldsymbol{U}_{\alpha}^{-}(2) \\
\boldsymbol{U}_{\beta}^{-}(2)
\end{array}\right],} \\
& {\left[\begin{array}{l}
\boldsymbol{U}_{\alpha}^{+}(2) \\
\boldsymbol{U}_{\beta}^{+}(2)
\end{array}\right]=\left[\begin{array}{ll}
\mathcal{Q}_{\alpha \alpha}^{++} & \mathcal{Q}_{\alpha \beta}^{++} \\
\mathcal{Q}_{\beta \alpha}^{++} & \mathcal{Q}_{\beta \beta}^{++}
\end{array}\right]\left[\begin{array}{l}
\boldsymbol{U}_{\alpha}^{+}(1) \\
\boldsymbol{U}_{\beta}^{+}(1)
\end{array}\right]+\left[\begin{array}{ll}
\mathcal{Q}_{\alpha \alpha}^{+-} & \mathcal{Q}_{\alpha \beta}^{+-} \\
\mathcal{Q}_{\beta \alpha}^{+-} & \mathcal{Q}_{\beta \beta}^{+-}
\end{array}\right]\left[\begin{array}{l}
\boldsymbol{U}_{\alpha}^{-}(2) \\
\boldsymbol{U}_{\beta}^{-}(2)
\end{array}\right],}
\end{aligned}
$$

where

$$
\begin{aligned}
\mathcal{Q}_{\kappa \kappa^{\prime}}^{s s^{\prime}} & =\Lambda_{\kappa}^{s} \Lambda_{\kappa^{\prime}}^{s^{\prime}} \delta_{\kappa \kappa^{\prime}} \delta_{s s^{\prime}}+\Lambda_{\kappa}^{s} M_{\kappa \kappa^{\prime}}^{s s^{\prime}} \Lambda_{\kappa^{\prime}}^{s^{\prime}}, \\
\Lambda_{\kappa}^{s} & =\operatorname{diag}\left(\exp \left(i s k_{\kappa \mathrm{g}_{1} z}^{s} a\right), \exp \left(i s k_{\kappa \mathrm{g}_{2} z}^{s} a\right), \ldots \exp \left(i s k_{\kappa \mathrm{g}_{N} z}^{s} a\right)\right) .
\end{aligned}
$$

For convenience of use in the following, Eqs. (10) and (11) can be rewritten as

$$
\begin{aligned}
& \boldsymbol{U}^{-}(1)=\boldsymbol{R}^{-+} \boldsymbol{U}^{+}(1)+\boldsymbol{T}^{--} \text {(1) } \boldsymbol{U}^{-} \text {(2), } \\
& \boldsymbol{U}^{+}(2)=\boldsymbol{T}^{++} \boldsymbol{U}^{+} \text {(1)+ } \boldsymbol{R}^{+-} \text {(1) } \boldsymbol{U}^{-} \text {(2). }
\end{aligned}
$$

Because the energy flux of the longitudinal wave and the transverse wave can be, respectively, estimated by

$$
\begin{aligned}
& q_{l}=\rho c_{l}^{2} \omega\left|\boldsymbol{U}_{\alpha \mathrm{g}}\right|^{2} k_{\alpha \mathrm{g}}, \\
& q_{t}=\rho c_{t}^{2} \omega\left|\boldsymbol{U}_{\beta \mathrm{g}}\right|^{2} k_{\beta \mathrm{g}},
\end{aligned}
$$

the transmission and reflection coefficients of a single layer of spheres can be estimated by

$$
T(R)=\frac{\sum_{\mathrm{g}}\left[c_{l}^{2}\left|\boldsymbol{U}_{\alpha \mathrm{g}}^{t r(r e f)}\right|^{2} k_{a \mathrm{~g} z}^{ \pm}+c_{t}^{2}\left|\boldsymbol{U}_{\beta \mathrm{g}}^{t r(r e f)}\right|^{2} k_{\beta \mathrm{g} z}^{ \pm}\right]}{\sum_{\mathrm{g}}\left[c_{l}^{2}\left|\boldsymbol{U}_{\alpha \mathrm{g}}^{i n}\right|^{2} k_{a \mathrm{~g} z}^{i n+}+c_{t}^{2}\left|\boldsymbol{U}_{\beta \mathrm{g}}^{i n}\right|^{2} k_{\beta \mathrm{g} z}^{i n+}\right]} .
$$

Now, let us formulate the reflection and transmission of multilayers of spheres by using the polymerization method. For the sake of clarity, we use $\boldsymbol{T}_{1}^{p q}$ and $\boldsymbol{R}_{1}^{p q}$ to represent the transmission and the reflection matrices 


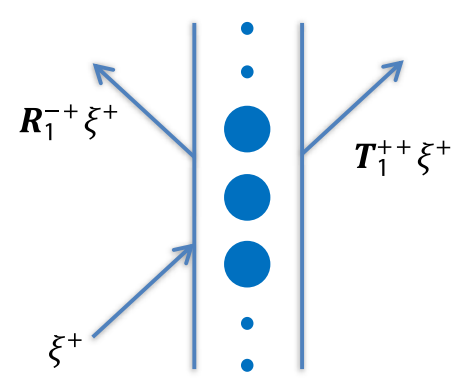

(a)

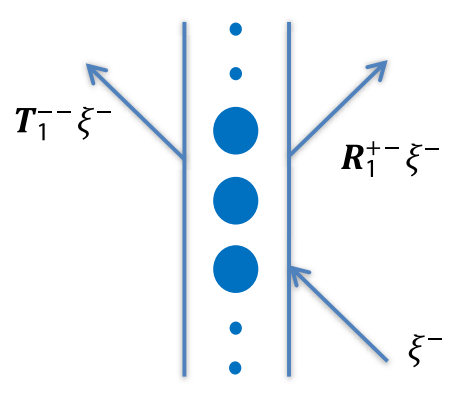

(b)

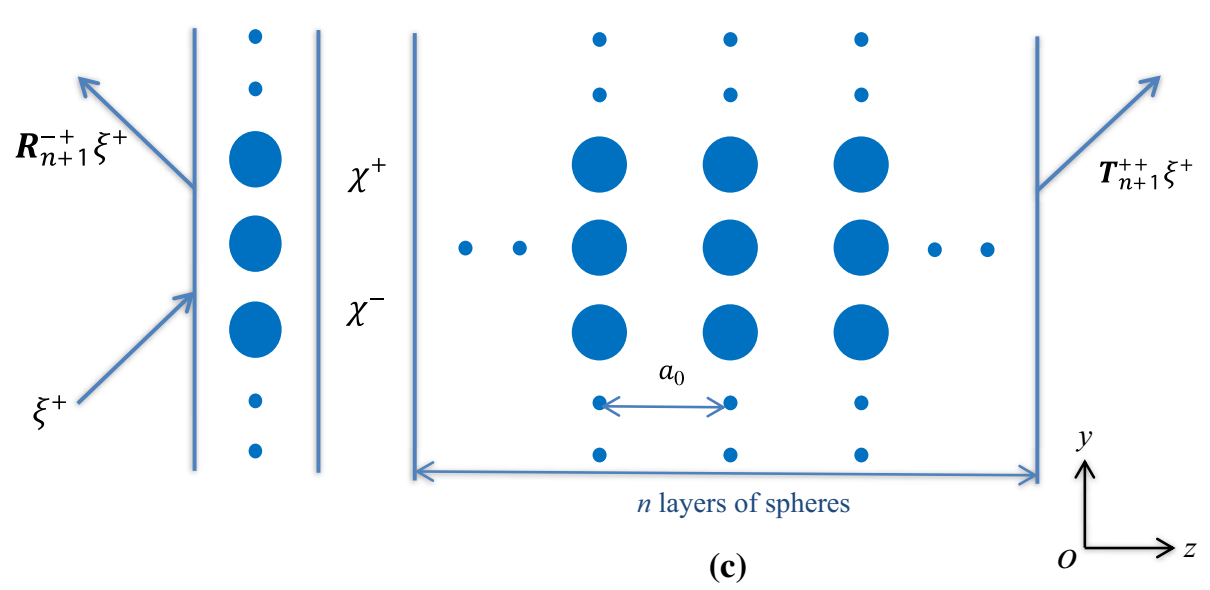

Fig. 1 The reflection and transmission through multilayers of spheres. a Reflection and transmission by a single layer of spheres when the incident wave is from the left; $\mathbf{b}$ reflection and transmission by a single layer of spheres when the incident wave is from the right; $\mathbf{c}$ reflection and transmission of $(n+1)$ layers of spheres

of a single layer of spheres; $\boldsymbol{T}_{n}^{p q}$ and $\boldsymbol{R}_{n}^{p q}$ to represent the transmission and the reflection matrices of $n$ layers of spheres; $\xi^{+}\left(\xi^{-}\right)$to represent an incident plane wave on the single layer of spheres from the left (right); and $\chi^{+}\left(\chi^{-}\right)$to represent the plane wave traveling from left (right) to right (left) between the single layer of spheres and the $n$ layers of spheres, see Fig. 1. The transmission wave and the reflection wave of the single layer of spheres for a left (right) incident wave $\xi^{+}\left(\xi^{-}\right)$can be expressed as $\boldsymbol{T}_{1}^{++} \xi^{+}\left(\boldsymbol{T}_{1}^{--} \xi^{-}\right)$and $\boldsymbol{R}_{1}^{-+} \xi^{+}\left(\boldsymbol{R}_{1}^{+-} \xi^{-}\right)$, respectively. Correspondingly, the transmission and reflection waves of $(n+1)$ layers of spheres can be expressed as $\boldsymbol{T}_{n+1}^{++} \xi^{+}$and $\boldsymbol{R}_{n+1}^{-+} \xi^{+}$for a left incident wave $\xi^{+}$. Then, the displacement field at two sides of a slab with $(n+1)$ layers of spheres can be obtained by a polymerization approach, namely

$$
\begin{aligned}
\boldsymbol{U}(1) & =\boldsymbol{R}_{n+1}^{-+} \xi^{+}=\boldsymbol{R}_{1}^{-+} \xi^{+}+\boldsymbol{T}_{1}^{--} \chi^{-}, \\
\boldsymbol{U}(2) & =\boldsymbol{T}_{n+1}^{++} \xi^{+}=\boldsymbol{T}_{n}^{++} \chi^{+}, \\
\chi^{+} & =\boldsymbol{T}_{1}^{++} \xi^{+}+\boldsymbol{R}_{1}^{+-} \chi^{-}, \\
\chi^{-} & =\boldsymbol{R}_{n}^{-+} \chi^{+} .
\end{aligned}
$$

Equation (16) means that the reflection wave field of the $(n+1)$ layers of spheres consists of two parts. One is the reflection wave field of the single layer of spheres triggered by the left incident wave field $\xi^{+}$, and the other is the transmission wave field of the single layer of spheres triggered by the right incident wave field $\chi^{-}$. Equation (17) means that the transmission wave field of the $(n+1)$ layers of spheres is triggered by the left incident wave field $\chi^{+}$impinged on the $n$ layers of spheres. Equation (18) means that the wave field $\chi^{+}$ consists of two parts. One is the transmission wave field of the single layer of spheres triggered by the left incident wave field $\xi^{+}$, and the other is the reflection wave field of the single layer of spheres triggered by the right incident wave field $\chi^{-}$. Equation (19) means that the wave field $\chi^{-}$is the reflection wave field of the $n$ layers of spheres triggered by the left incident wave field $\chi^{+}$. Eliminating the $\chi^{+}$and $\chi^{-}$of Eqs. (16-19), the 
iterative formulas of the transmission and reflection matrix of $(n+1)$ layer of spheres are obtained:

$$
\begin{aligned}
& \boldsymbol{T}_{n+1}^{++}=\boldsymbol{T}_{n}^{++}\left(\boldsymbol{I}-\boldsymbol{R}_{1}^{+-} \boldsymbol{R}_{n}^{-+}\right)^{-1} \boldsymbol{T}_{1}^{++} \\
& \boldsymbol{R}_{n+1}^{-+}=\boldsymbol{R}_{1}^{-+}+\boldsymbol{T}_{1}^{--} \boldsymbol{R}_{n}^{-+}\left(\boldsymbol{I}-\boldsymbol{R}_{1}^{+-} \boldsymbol{R}_{n}^{-+}\right)^{-1} \boldsymbol{T}_{1}^{++} .
\end{aligned}
$$

From Eqs. (20) and (21), the transmission and reflection matrices of any multilayers of spheres can be obtained by repeating the polymerization process when the transmission and reflection matrices of every single layer, namely $\boldsymbol{T}_{1}^{p q}$ and $\boldsymbol{R}_{1}^{p q}$, are given. Then, inserting $\boldsymbol{U}(1)=\boldsymbol{R}_{n+1}^{-+} \xi^{+}$and $\boldsymbol{U}(2)=\boldsymbol{T}_{n+1}^{++} \xi^{+}$into Eq. (15), the reflection and transmission coefficients of any multilayers of spheres can be calculated.

\section{Numerical results and discussion}

In the numerical examples, the periodic composites are composed of 15 layers of steel spheres embedded in a polyester host matrix. The material parameters of steel sphere are $\rho=7800\left(\mathrm{~kg} \mathrm{~m}^{-3}\right), c_{l}=5940\left(\mathrm{~ms}^{-1}\right), c_{t}=$ $3200\left(\mathrm{~ms}^{-1}\right), r=0.25 a_{0}$, and the material parameters of polymer host are $\rho=1220\left(\mathrm{~kg} \mathrm{~m}^{-3}\right), c_{l}=$ $2490\left(\mathrm{~ms}^{-1}\right), c_{t}=1180\left(\mathrm{~ms}^{-1}\right)$. The steel spheres are arranged in a hexagonal lattice in the plane parallel to the plane $o x y$, and the lattice constant is $a_{0}$, see Fig. 2. Fifteen layers of spheres are arranged parallel and aligned to each other, and the spacing between two adjacent layers is $d=a_{0}$.

Figure 3 shows the transmission coefficients of normal incident longitudinal and transverse waves. It is observed that there is a frequency range which extends from the normalized frequency $\omega a_{0} / c_{0}=2.05$ up to

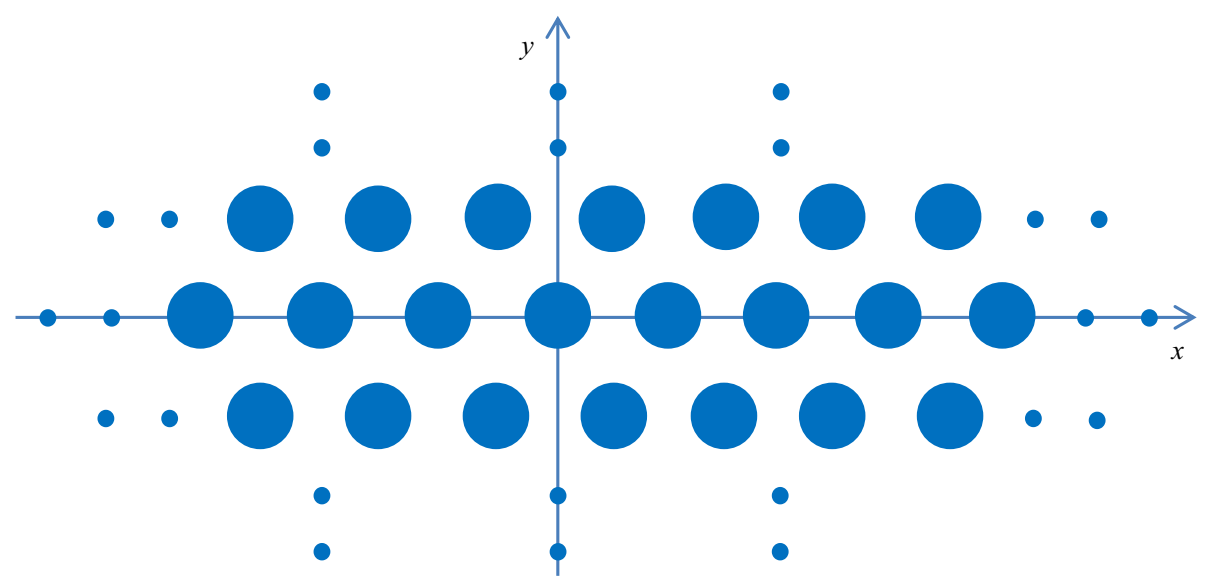

Fig. 2 Sphere layers parallel to the $x y$-plane with spherical scatterers arranged on sites of the hexagonal lattice
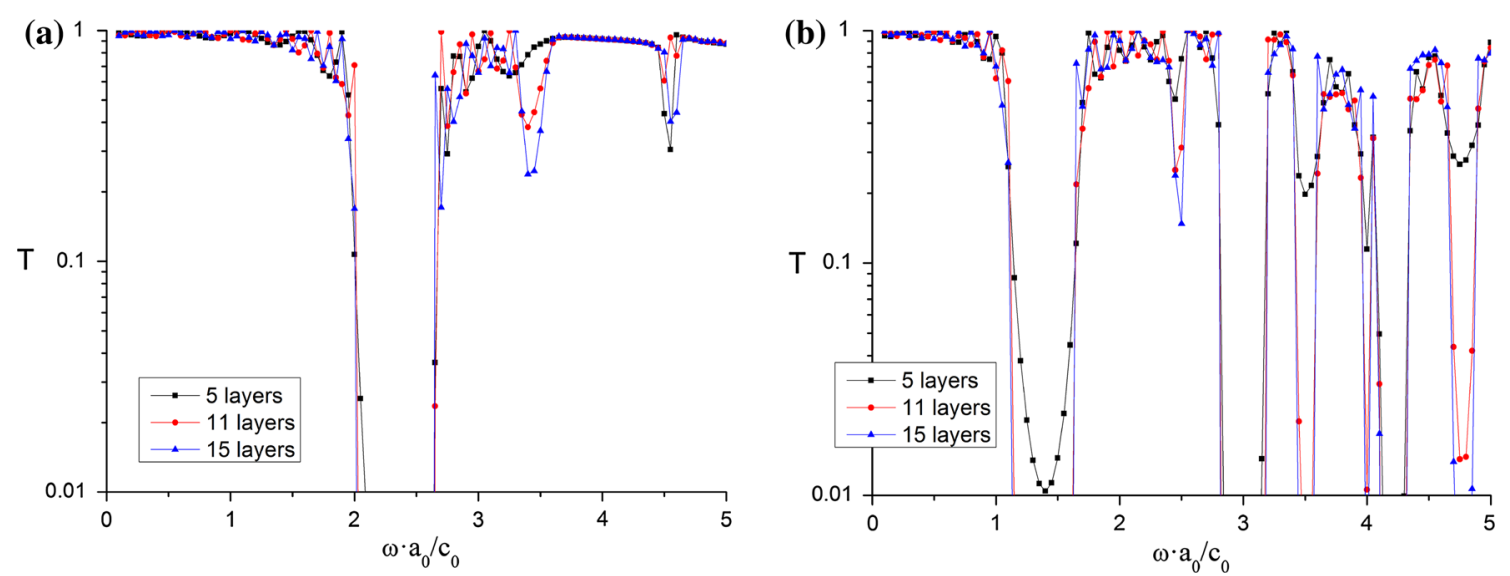

Fig. 3 The transmission coefficients at different frequency of normal incident wave. a Incident longitudinal wave; $\mathbf{b}$ incident transverse wave 

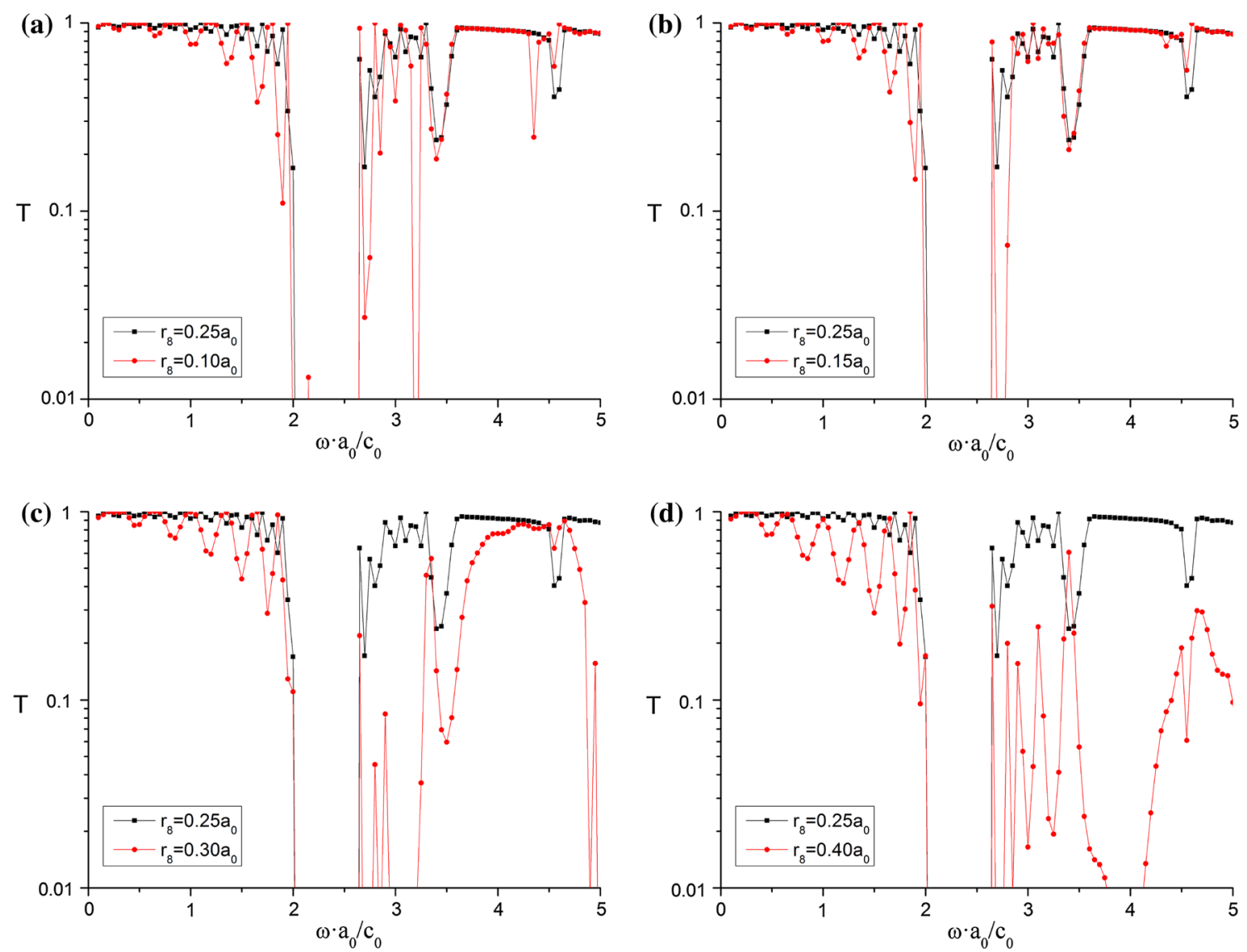

Fig. 4 The influences on transmission coefficients and the defect states of one planar defect layer created by changing the radiuses of scatterers of the eighth layer of spheres ( $c_{0}$ is the speed of the longitudinal wave in the host medium). $\mathbf{a} r_{8}=0.10 a_{0}$; $\mathbf{b} r_{8}=0.15 a_{0} ; \mathbf{c} r_{8}=0.35 a_{0} ; \mathbf{d} r_{8}=0.40 a_{0}$. The radius of spheres in other layers is $0.25 a_{0}$

$\omega a_{0} / c_{0}=2.65$ in the transmission curves of longitudinal incident wave, and within this range, the transmission coefficients are nearly zero. This frequency band with nearly zero transmission corresponds to the forbidden band of an infinite periodical structure. Its width may differ for different number of layers of spheres, but 15 layers of spheres already have a good convergence which is asymptotic to the forbidden band of an infinite periodic structure. As similar phenomenon is observed for the transmission curves of incident transverse waves. However, there is more than one forbidden band observed in the frequency range considered. The first forbidden band extends from the normalized frequency $\omega a_{0} / c_{0}=1.15$ up to $\omega a_{0} / c_{0}=1.60$, and the second forbidden band extends from the normalized frequency $\omega a_{0} / c_{0}=2.85$ up to $\omega a_{0} / c_{0}=3.15$. It is known that the forbidden band is the unique property of the periodic structure. The introduction of defects will result in the destruction of periodicity and therefore will impose influences on the forbidden band evidently.

Figure 4 shows the influences of one planar defect on the transmission curves. The planar defect is created by changing the radiuses of spheres in the eighth layer. Such a planar defect in the multiple layers of spheres results in one or multiple transmission resonances that appear at a certain frequency within the band gap. These transmission resonances signify the existence of modes of vibration of the elastic field that extend to infinity parallel to the surface of the slab in the manner of a Bloch wave, but decay rapidly normal to the planar defect on either side of it. Such a state of the elastic field centered on the planar defect is usually called defect state. It is observed that there is only one defect state appearing when the radius of the identical spheres in the eighth layer is smaller than that in other layers. But there are more than one defect states appearing when the radius of identical spheres in the eighth layer is greater than that in other layers. Moreover, the introduction of planar defect makes the width of the band gap evidently wider. The lower edge has no notable shift, but the upper edge shifts evidently toward a high frequency when the radius of the spheres in the eighth layer increases gradually. This results in the wider band gap. 

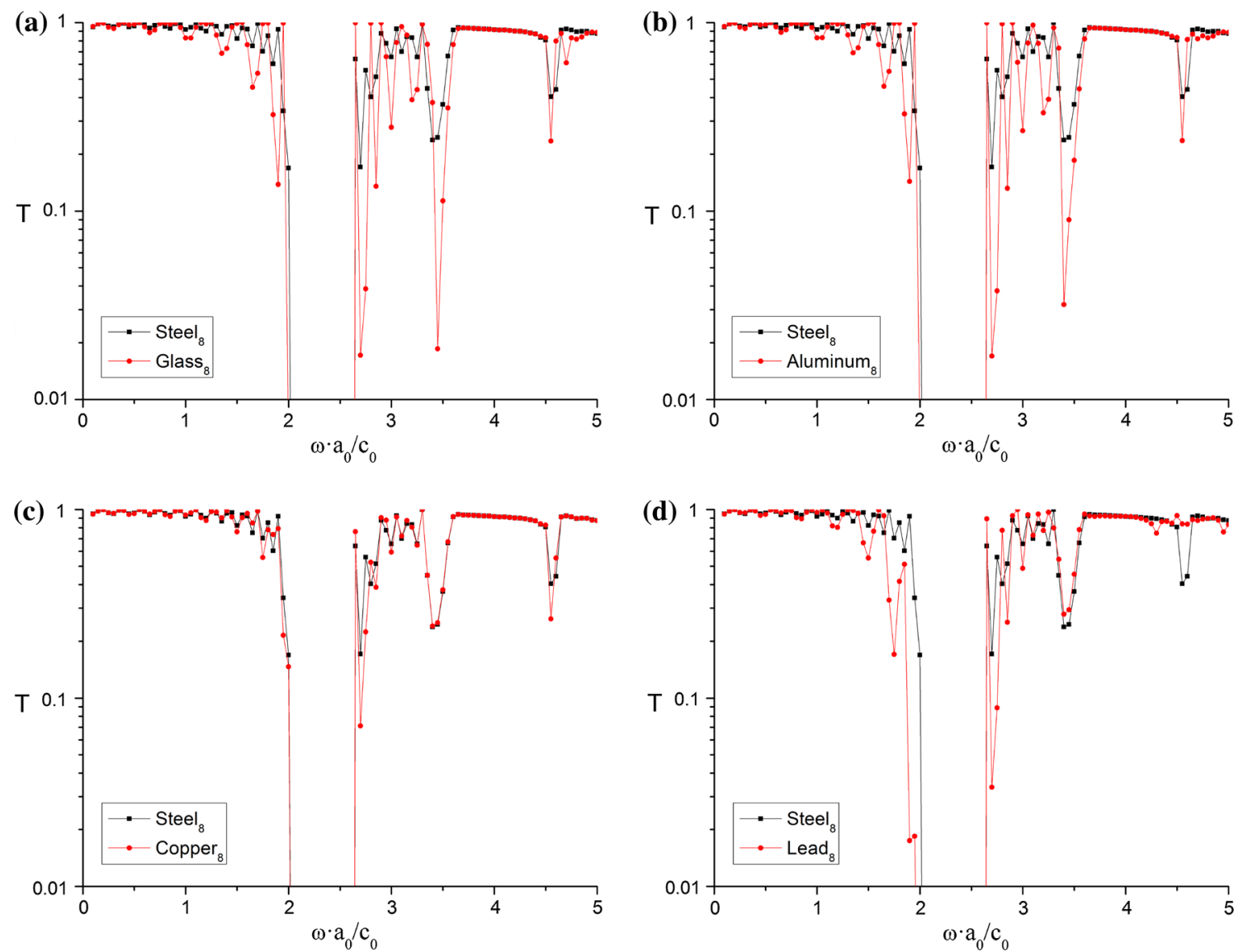

Fig. 5 The influences of one planar defect layer created by replacing steel spheres of the eighth layer by a glass spheres; $\mathbf{b}$ aluminum spheres; c copper spheres; d lead spheres

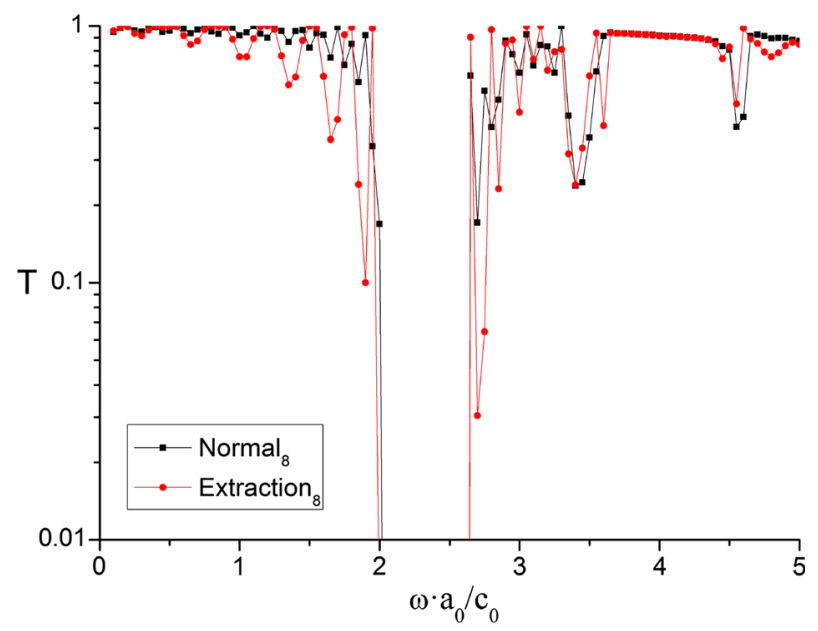

Fig. 6 The influences of one planar defect layer created by removing the eighth sphere layer

Figure 5 also shows the influences of one planar defect on the transmission curves. But the planar defect is formed by replacing the steel spheres in the eighth layer with the glass spheres, aluminum spheres, copper spheres and lead spheres, respectively. In contrast, the influences of one planar defect formed by changing the physical property of spheres are much smaller than of one formed by changing the radius of spheres. The only noticeable change takes place when the steel spheres in the eighth layer are replaced by the heavier lead 

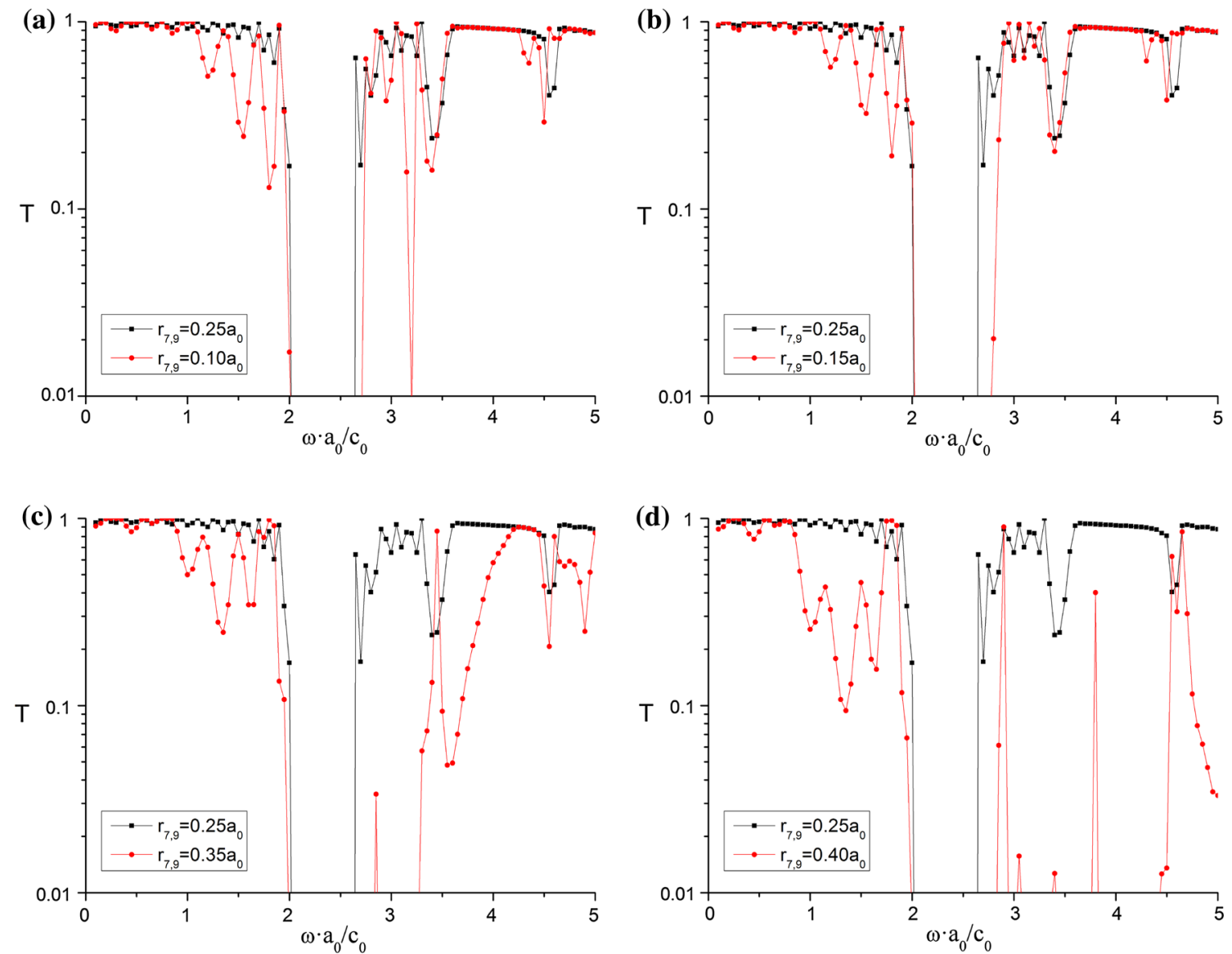

Fig. 7 The influences of two symmetrical planar defect layers created by changing the radiuses of scatterers of the seventh and the ninth layers. a $r_{7,9}=0.10 a_{0} ; \mathbf{b} r_{7,9}=0.15 a_{0} ; \mathbf{c} r_{7,9}=0.35 a_{0} ; \mathbf{d} r_{7,9}=0.40 a_{0}$. The radius of spheres in other layers is $0.25 a_{0}$

spheres. In this situation, the lower edge of the band gap shifts toward a low frequency and thus the band gap is widened slightly.

Figure 6 shows the influences on transmission curves by another kind of planar defect, namely when the spheres in the eighth layer are removed. It is observed that the lower edge of band gap shifts toward a lower frequency slightly and the width of the band gap does not change noticeably. By comparing Figs. 4, 5 and 6, it is found that the planar defect formed by changing the radius of spheres is the most effective for creating defect states among the three kinds of planar defects considered in this paper. Only the planar defect formed by changing the radius of spheres can widen the band gap evidently and results in the appearance of defect states. Such a structure can be designed elaborately to be used as narrow band-pass acoustical filters.

After the influences of one planar defect are known, we still want to know what will happen when more than one planar defect is introduced. In order to answer this question, we further studied the influences of two planar defects located symmetrically on two sides of the eighth layer. The two planar defects are formed by changing the radiuses of two layers of spheres at the same time. Our main concerns focus on the change of the transmission curves when the distance between the two planar defects increases gradually.

Figure 7 shows the transmission curves when two planar defect layers are located at the seventh and the ninth layers, respectively. The distance between the two planar defect layers is $2 a_{0}$ in this situation. It is found that there are no defect states appearing in the original band gap when the radius of identical spheres in the defect layers is smaller than that in the other layers (the radius of spheres in the other layers is $0.25 a_{0}$.). There is one defect state appear when the radius of spheres in the defect layers increases to $r_{7,9}=0.35 a_{0}$, while two evident defect states appear when the radius of spheres in the defect layers increases to $r_{7,9}=0.4 a_{0}$. Figure 8 shows the transmission curves when two planar defect layers are located at the fifth and the eleventh layers, respectively. The distance between the two planar defect layers becomes $6 a_{0}$ in this situation. It is found that 

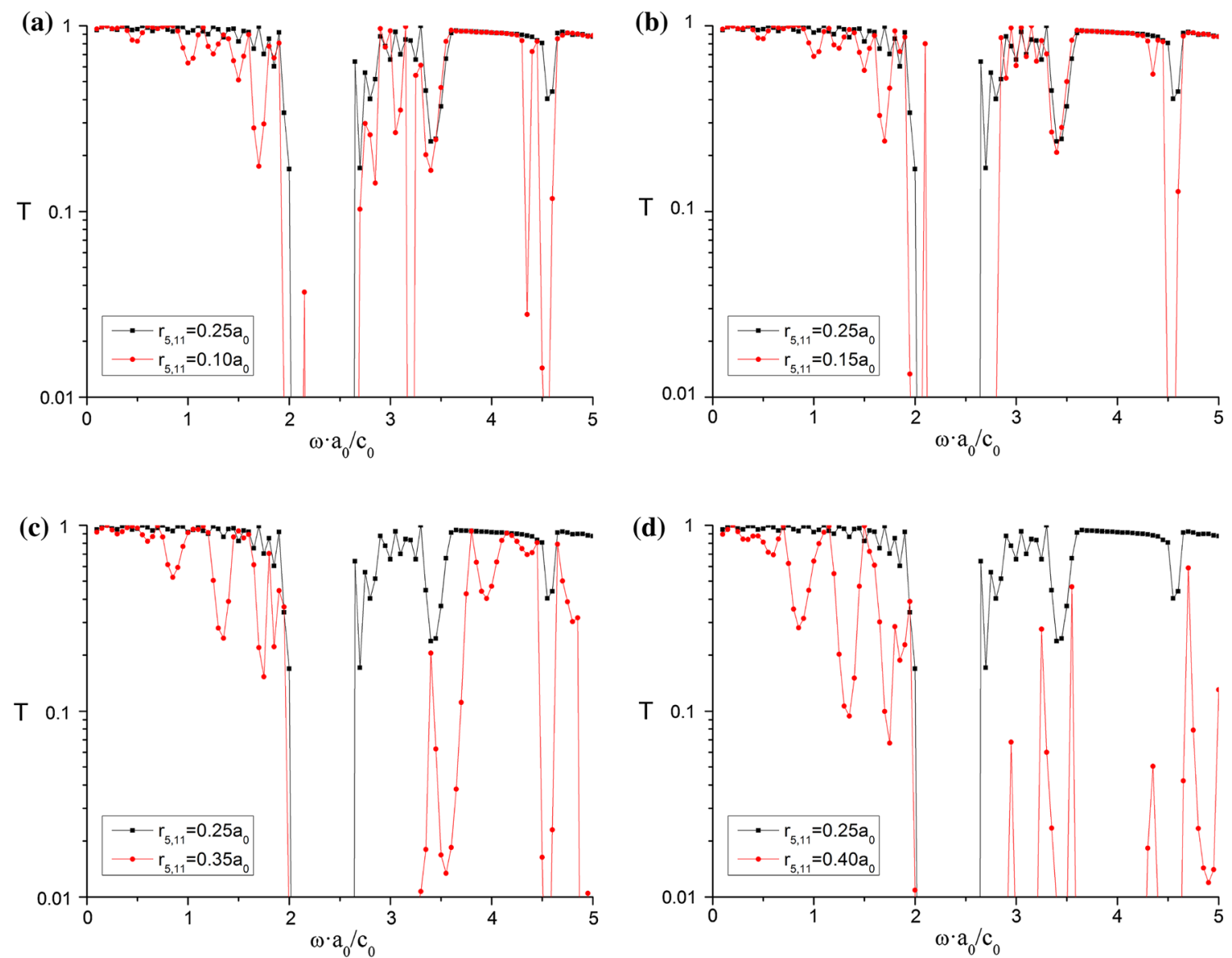

Fig. 8 The influences of two symmetrical planar defects created by changing the radiuses of scatterers of the fifth and the eleventh layers. a $r_{5,11}=0.10 a_{0} ; \mathbf{b} r_{5,11}=0.15 a_{0} ; \mathbf{c} r_{5,11}=0.35 a_{0} ; \mathbf{d} r_{5,11}=0.40 a_{0}$. The radius of spheres in other layers is $0.25 a_{0}$

there is one defect state appearing in the original band gap when the radius of spheres in the defect layers is smaller than that in the other layers. When the radius of spheres in the defect layers increases to $r_{5,11}=0.4 a_{0}$, there are four evident defect states appearing. This observation means that the bigger distance between two planar defect layers can make the defect states appearing more easily. This is due to the extra interference between two planar defect layers.

Apart from two symmetrical defect layers, the case of asymmetrical arrangement of two defect layers is also interesting. In order to investigate the case, two defect layers are asymmetrically arranged at the seventh and the eleventh layers instead of at the seventh and the ninth layers. Figure 9 shows the transmission curves in this situation. By comparing Figs. 7 and 8 with Fig. 9, it is found that asymmetrically arranging planar defects makes the defect states in Fig. 8a, b disappear and the defect states in Fig. 8d blurry. These observations signify that arranging planar defects asymmetrically does not help to enhance defect states.

\section{Conclusions}

There are some forbidden transmission bands where the transmission coefficients become nearly zero when the elastic waves propagate through periodically arranged multilayers of spheres. These forbidden transmission bands correspond to the stop band appearing in a perfect periodic structure. The introduction of defect layers will result in the destruction of periodicity and therefore activate the defect states (the narrow band of transmission within the stop band). The planar defect can be created by changing the radius or the material of spheres in one specific layer and even by removing one layer of spheres. However, it is found from the numerical simulations that changing the radius of identical spheres in one layer is the most effective way to create the defect states. A smaller radius of spheres in the defect layer contributes to the appearance of the lower frequency defect 

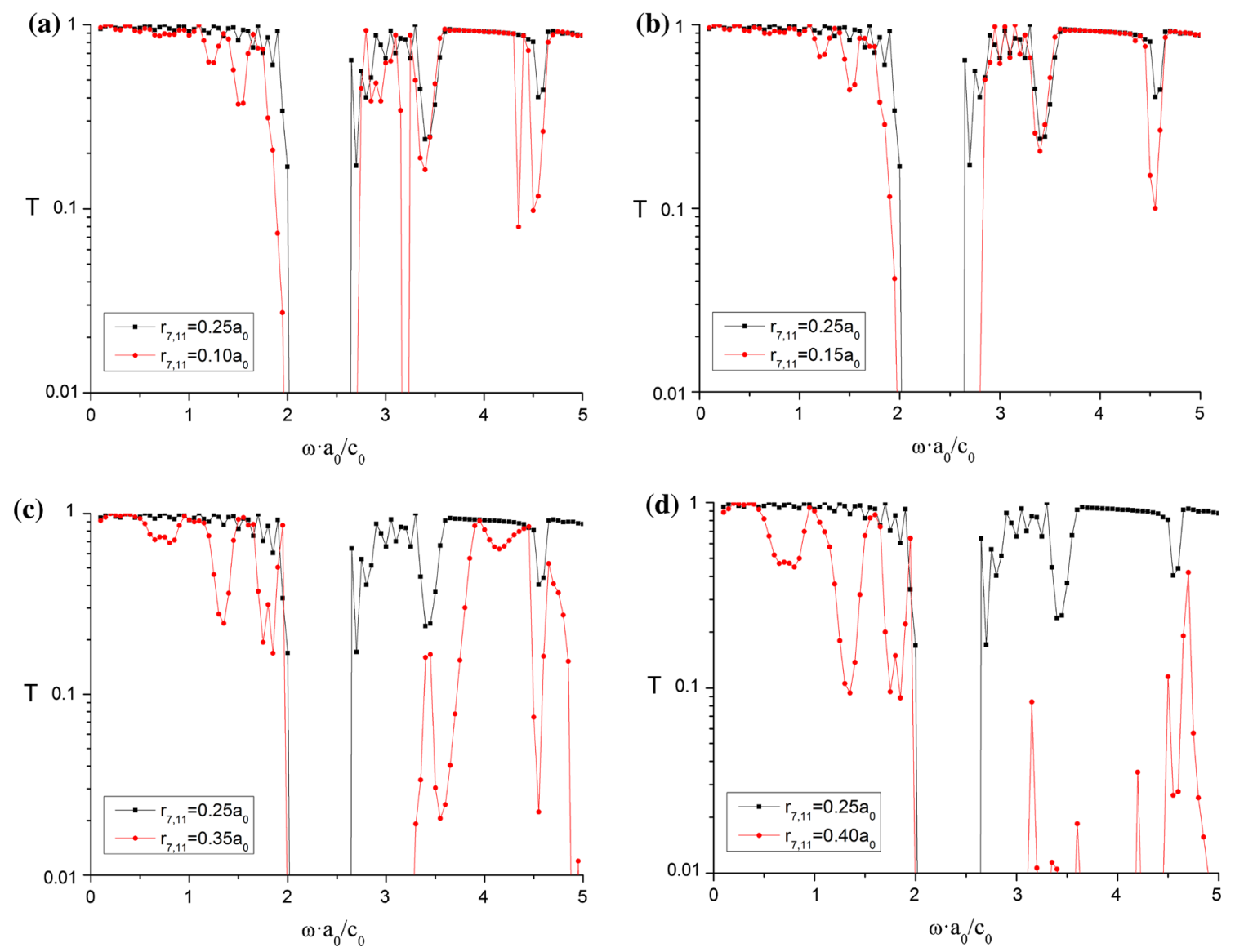

Fig. 9 The influences of two asymmetrical planar defects created by changing the radiuses of spheres in the seventh and the eleventh layers. a $r_{7,11}=0.10 a_{0} ; \mathbf{b} r_{7,11}=0.15 a_{0} ; \mathbf{c} r_{7,11}=0.35 a_{0} ; \mathbf{d} r_{7,11}=0.40 a_{0}$. The radius of spheres in other layers is $0.25 a_{0}$

states, but a larger radius of spheres contributes to the appearance of the higher-frequency defect states. Two planar defect layers with symmetrical arrangement can further enhance the number and the quality of defect states which appear in the stop band of the perfect periodical structure. The asymmetrical arrangement of two planar defect layers does not help the creation of defect states and the improvement in quality of the existed defect states. The existence of defect states signifies that it should be possible to produce extremely narrow band filters by the introduction of different kinds of planar defects in the periodical structure. The present study provides some useful information for the design of specific acoustical filters by the use of periodically arranged multilayers of spheres with elaborately arranged planar defects.

Acknowledgments The work is supported by the National Natural Science Foundation of China (No. 10972029) and Opening Fund of State Key Laboratory of Nonlinear Mechanics (LNM).

\section{References}

1. Liu, Z.Y., Chan, C.T., Sheng, P.: Elastic wave scattering by periodic structures of spherical objects: theory and experiment. Phys. Rev. B 62, 2446-2457 (2000)

2. Psarobas, I.E., Stefanou, N., Modinos, A.: Scattering of elastic waves by periodic arrays of spherical bodies. Phys. Rev. B 62(1), 278-291 (2000)

3. Liu, Z., Chan, C.T., Sheng, P.: Three-component elastic wave band-gap material. Phys. Rev. B 65(16), 165116 (2002)

4. Sainidou, R., Stefanou, N., Psarobas, I.E. et al.: Scattering of elastic waves by a periodic monolayer of spheres. Phys. Rev. B 66(2), 024303 (2002)

5. Sainidou, R., Stefanou, N., Psarobas, I.E., Modinos, A.: A layer-multiple-scattering method for phononic crystals and heterostructures of such. Comput. Phys. Commun. 166, 197-240 (2005) 
6. Wei, P.J., Huang, Z.P.: Dynamic effective properties of the particle-reinforced composites with the viscoelastic interphase. Int. J. Solids Struct. 41(24-25), 6993-7007 (2004)

7. Wei, P.J.: A self-consistent approach to the dynamic effective properties of composites reinforced by distributed spherical particles. Acta Mech. 185, 67-79 (2006)

8. Fang, X.Q., Wang, D.B., Liu, J.X.: Multiple scattering of elastic waves in metal-matrix composite materials with high volume concentration of particles. Eur. J. Mech. A/Solids 28, 377-386 (2009)

9. James, R., Woodley, S.M., Dyer, C.M. et al.: Sonic bands, bandgaps, and defect states in layered structures-theory and experiment. J. Acoust. Soc. Am. 97(4), 2041-2047 (1995)

10. Munday, J.N., Bennett, C.B., Robson, W.M.: Band gaps and defect modes in periodically structured waveguides. J. Acoust. Soc. Am. 112(4), 1353-1358 (2002)

11. Robertson, W.M., Baker, C., Bennett, C.B.: Slow group velocity propagation of sound via defect coupling in a onedimensional acoustic band gap array. Am. J. Phys. 72(2), 255-257 (2004)

12. Sigalas, M.M.: Elastic wave band gaps and defect states in two-dimensional composites. J. Acoust. Soc. Am. 101(3), 1256$1261(1997)$

13. Sigalas, M.M.: Defects states of acoustic waves in a two-dimensional lattice of solid cylinders. J. Appl. Phys. 84(6), 30263030 (1998)

14. Torres, M., Montero de Espinosa, F.R., García-Pablos, D. et al.: Sonic band gaps in finite elastic media: Surface states and localization phenomena in linear and point defects. Phys. Rev. Lett. 82(15), 3054-3057 (1999)

15. Kafesaki, M., Sigalas, M.M., García, N.: Frequency modulation in transmittivity of wave guides in elastic-wave band-gap materials. Phys. Rev. Lett. 85(19), 4044-4047 (2000)

16. Kafesaki, M., Sigalas, M.M., García, N.: Wave guides in two-dimensional elastic wave band gap materials. Phys. B 296 (1-3), 190-194 (2001)

17. Wu, F., Hou, Z., Liu, Z. et al.: Point defect states in two-dimensional phononic crystals. Phys. Lett. A 292(3), 198-202 (2001)

18. Wu, F.G., Liu, Z.Y., Liu, Y.Y.: Stop gaps and single defect states of acoustic waves in two-dimensional lattice of liquid cylinders. Chin. Phys. Lett. 18(6), 785-787 (2001)

19. Wu, F., Liu, Z., Liu, Y.: Splitting and tuning characteristics of the point defect modes in two-dimensional phononic crystals. Phys. Rev. E 69(6), 066608 (2004)

20. Chandra, H., Deymier, P.A., Vasseur, J.O.: Elastic wave propagation along waveguides in three-dimensional phononic crystals. Phys. Rev. B 70(5), 054302 (2004)

21. Pasrobas, I.E., Stefanou, N., Modinos, A.: Phononic crystals with planar defects. Phys. Rev. B 62(9), 5536-5540 (2000)

22. Zhao, Q., Wei, P.J.: The reflection and transmission of elastic waves through a plane of spheres in periodic arrangement. Acta Mech. 224, 1009-1018 (2013) 